\title{
ANGIOGENESIS PROCESS WITH VESSEL IMPAIRMENT FOR GOMPERTZIAN AND LOGISTIC TYPE OF TUMOUR GROWTH
}

Abstract. We propose two models of vessel impairment in the process of tumour angiogenesis and we consider three types of treatment: standard chemotherapy, antiangiogenic treatment and a combined treatment. The models are based on the idea of Hahnfeldt et al. that the carrying capacity for any solid tumour depends on its vessel density. In the models proposed the carrying capacity also depends on the process of vessel impairment. In the first model a logistic type equation is used to describe the neoplastic cell dynamics, while in the second one we use the Gompertz type of growth. Simulations of solutions show that a vascular dormant state of the tumour can be reached in two different ways. In addition in each case efficiency of treatments is different.

1. Introduction. In adults, the normal physiological role of angiogenesis - the process of new vessel formation - is restricted to wound healing, menstrual cycle and pregnancy. In addition, angiogenesis is critical during fetal development. Unfortunately, it is also essential to the growth and development of solid tumours. After reaching an avascular dormant state, a tumour can grow further only by inducing vessels in host tissue to sprout capillary tubes which migrate towards and ultimately penetrate the tumour, providing it with a circulating blood supply and, therefore, an additional source of nutrients [20,21].

Despite the essential role of angiogenesis in tumour growth, it has been discovered that tumour angiogenesis is highly pathological. Incorrect struc-

2000 Mathematics Subject Classification: 34A12, 34A34, 92C50.

Key words and phrases: tumour, angiogenesis, Hahnfeldt et al. model, vessel impairment, chemotherapy, antiangiogenic treatment, ordinary differential equations, steady state, invariant set. 
ture and poor efficiency of newly formed vessels are common tumour features $[20,21]$. In some experiments to investigate tumour biology it has been shown that most of the administered dose of chemotherapy was not even absorbed by the tumour. Moreover, the absorbed part was not evenly distributed in different tumour regions. This makes effective treatment difficult, because cells which do not get sufficient amount of drug can survive and even if they are only few, repeated tumour growth is inevitable.

Healthy tissues are nourished by straight vessels, which ramify in a predictable way to smaller ones and at the end to capillary tubes. Vessels which were build due to tumour stimuli are instead arranged in tangled knots. They connect with each other in a random way, some of their branches are excessively big, there appear additional immature capillary tubes, or what can be even worse, they do not exist in some tumour regions. In addition tumour vessels work poorly, because they are build incorrectly. It has been discovered that in some vessels the blood stream is excessively rapid, in others excessively slow and in some of them it turns back periodically. This makes even distribution of drugs very difficult. In addition, some sections of vessel walls are poorly permeable, whereas others are very leaky. This is caused by incorrect structure of pores in vessel walls, which can have diameter almost one hundred times larger than in healthy tissue. Hence, it is almost impossible to maintain correct pressure gradient, which is essential in efficient exchange of oxygen, nutrient and drugs between vessel and cells. This also causes an increase of interstitial pressure, which may lead to necrosis in some tumour regions.

It has been experimentally confirmed that pathological angiogenesis is caused by long lasting overexpression of proangiogenic factors, like e.g. VEGF, vascular endothelial growth factor [20,21]. In healthy tissue proangiogenic factors are balanced by natural inhibitors, like e.g. trombospodin.

Influencing the process of angiogenesis is currently one of the most important methods in cancer treatment. This method was proposed by Folkman [13]. However, its implementation was possible due to the discovery of anti-angiogenic drugs by O'Reilly et al. [30, 31] (see also [14, 15]). This method owes its effectiveness to a very small group of substances that cause formation of new blood vessels. Thus, it is easier to create universal drugs to fight cancer. Increased survivorship of patients in the case when chemotherapy and antiangiogenic therapy were combined was observed in clinical trials. An explanation is that dosing angiogenic inhibitors causes normalization of tumour vasculature; then chemotherapy reaches optimal effectiveness. Previously some studies incorporated mathematical models for the development of tumour under angiogenic signalling: see the review in [28] and references therein or [8], where also other processes connected with tumour growth are presented. 
The most important model from the biological point of view was proposed by Hahnfeldt et al. in [19]. Its importance is caused by the biological validity confirmed by lab experiments. On the basis of the general idea of this model, a slightly different one was formulated in [12], where also the optimal scheduling of radiotherapy and angiogenic inhibitors was proposed. A family of models based on the Hahnfeldt et al. model is an object of study of several groups of researchers. We should mention d'Onofrio and Gandolfi $[9,10,11]$, who studied such models from the mathematical point of view and from the point of view of anti-angiogenic therapy, while in the papers of Świerniak and Świerniak et al. [33-35] optimal control problems for the models were formulated and analysed. Slightly different control problems for the angiogenesis process and anti-angiogenic therapy were studied by Ledzewicz and Schättler [24-27]. On the other hand, in the literature we can also find models built on different assumptions, like those proposed in [3, 2], and models combining different approaches to angiogenesis (see e.g. [6]).

In this paper, following the ideas presented in [32] we propose two qualitative models for tumour growth under angiogenic stimulator/inhibitor control that includes vascular impairment. On the basis of these models, we investigate effects of therapy that combines administration of angiogenic inhibitors and chemotherapy.

2. Model presentation. According to clinical and laboratory observations, usage of Gompertz [18] (cf. also [22, 23, 4, 5, 36]) or logistic type (cf. e.g. $[16,5,36,29]$ ) equations in modelling of avascular tumour growth gives good qualitative results. It illustrates well the empirically observed phenomenon that a tumour without vasculature grows only to a finite size (about 2-3 $\mathrm{mm}$ of diameter). Let $T$ denote the total neoplastic cell mass. Let $T_{\max }$ be the environmental capacity of its growth, that is, the maximal mass till which the tumour without vasculature can grow. The logistic equation for $T$ has the form

$$
\dot{T}=\lambda_{1} T\left(1-\frac{T}{T_{\max }}\right),
$$

while the Gompertzian type of growth is described by the equation

$$
\dot{T}=-\beta_{1} T \ln \left(\frac{T}{T_{\max }}\right),
$$

where $\lambda_{1}$ and $\beta_{1}$ are the parameters that describe the dynamics of tumour growth in the respective equations. It should be noticed that in the original Hahnfeldt et al. model [19] of angiogenesis the Gompertzian type of growth was used. However, in the papers of d'Onofrio and Gandolfi (see e.g. [9]) or Swierniak (see [33]), the logistic type was used as well. In the following, 
instead of equation (1) or (2) we will use the general form

$$
\dot{T}=T h\left(\frac{T}{T_{\max }}\right),
$$

where $h$ is either linear (for (1)) or logarithmic (for (2)).

Following Hahnfeldt et al. [19], we assume that during angiogenesis the environmental capacity for neoplastic cell mass increases. Due to increased ability of supplying nutrients inside tumour, its further growth is possible. Let $V$ denote the mass of vessels that supply the tumour with additional nutrients. It is obvious that with the increase of $V$, the environmental limitation rises as well. However, vessel growth inside the tumour does not proceed as in a healthy tissue $[20,21,32]$. Prolonged lack of balance between angiogenic stimulators and inhibitors can yield progressive impairment of already formed vessels. The diameter of pores in vessel walls gets larger, which results in a rapid decrease of the diffusion process efficiency and a simultaneous increase of pressure inside the tumour. Thus, the entropy of the whole vessel structure increases, causing vascular walls to collapse.

Let $I$ denote the percentage of impaired vessels inside the tumour. According to the assumption that environmental limitation increases, (3) takes the form

$$
\dot{T}=T h\left(\frac{T}{T_{\max }+f(V, I)}\right),
$$

where $f(V, I) \geq 0$ (see [32]). To determine the form of the function $f$ let $\varepsilon_{1}>0$ be the coefficient of healthy vessel efficiency, and $\gamma_{1}>0$ the vessel efficiency decrease at a fixed level of vasculature impairment. Suppose that $\varepsilon_{1}>\gamma_{1}$, which reflects the fact that even if the impairment of vasculature reaches $100 \%$, the vessels still supply the tumour in some amount of nutrients. Therefore, as the environmental limitation is proportional to $V$ and we assume that the effectiveness of vasculature decreases with increasing impairment, let the equation for $T$ have the following form:

$$
\dot{T}=T h\left(\frac{T}{T_{\max }+V\left(\varepsilon_{1}-\gamma_{1} I\right)}\right) .
$$

Growth and decay of vessels are caused by lack of balance between angiogenic stimulators (e.g. VEGF, vascular endothelial growth factor) and natural inhibitors (e.g. angiostatin, trombospodin and endostatin) [20, 21]. Let $U$ denote the difference between the amount of proangiogenic factors and angiogenic inhibitors. Thus, when $U>0$, the process of vascular growth begins, and when $U<0$, the process of vascular decay begins. Suppose that when $U=0$, the amount of vessels does not change. Due to endothelial cell death or disaggregation, the amount of vessels always changes, but the process is so slow that it can be ignored in the model [6]. 
The rate of vessel growth is proportional to the magnitude of unbalance between stimulators and inhibitors. It is also proportional to the amount of already existing vessels, because new vessels are their new branches. It can also be assumed that the rate of vessel growth is decreased by the impairment of vasculature. Therefore, on that assumption, the equation for the rate of vessel mass change has the form

$$
\dot{V}=\lambda_{2} U V(1-I) \text {. }
$$

As I represents the proportion of impaired vessel amount to the total amount of vessels, it can only take values in $[0,1]$. Simultaneously, the dynamics of $I$ 's change is highest when its value is near $1 / 2$, because the probability of the impairment change is than the highest. Accordingly, the equation for $I$ has the form

$$
\dot{I}=g(T, V, U) I(1-I) .
$$

The rate of vasculature impairment change also depends on the level of unbalance between angiogenic enzymes [20,21]. The impairment starts increasing when proangiogenic unbalance appears, and its rate of change is proportional to its magnitude. On the other hand, vasculature starts to normalize when $U$ gets below zero. However, even when the level of unbalance is very high, at the beginning of angiogenesis, new vessels cannot get impaired immediately. Till some instance, even high unbalance causes only high rate of change in the vessel amount. Accordingly, the equation for $I$ takes the form

$$
\dot{I}=\lambda_{3} U\left(\frac{V}{T}\right)^{\alpha} I(1-I)
$$

where $\alpha \geq 1$.

Angiogenic stimulators and natural inhibitors are secreted by neoplastic cells and their surroundings. A larger neoplastic cell mass yields a larger amount of proangiogenic factors. Together with the vessel development, a larger amount of angiogenic inhibitors appears.

The rate of angiogenic factors secretion also depends on the level of vessel impairment. If $I$ is higher, the ability of angiogenic factors to penetrate the surroundings is compromised. Thus, we propose the following equation for $U$ :

$$
\dot{U}=\left(\lambda_{4} T-\lambda_{5} V\right)(1-I) .
$$

Combining equations (5)-(8), we propose the following system of four ODEs describing tumour development under angiogenic signalling with dependence on vessel impairment: 


$$
\left\{\begin{array}{l}
\dot{T}=T h\left(\frac{T}{T_{\max }+V\left(\varepsilon_{1}-\gamma_{1} I\right)}\right), \\
\dot{V}=\lambda_{2} U V(1-I), \\
\dot{I}=\lambda_{3} U\left(\frac{V}{T}\right)^{\alpha} I(1-I), \\
\dot{U}=\left(\lambda_{4} T-\lambda_{5} V\right)(1-I)
\end{array}\right.
$$

with positive coefficients and $\varepsilon_{1}>\gamma_{1}$. Let (9a) and (9b) denote the system (9) with the Gompertzian and logistic type of neoplastic cell growth, respectively.

2.1. Basic properties. We analyze equations (9) in the phase space $\mathcal{S}=$ $\{(T, V, I, U): T>0, V \geq 0, I \in[0,1], U \in \mathbb{R}\}$ assuming that all the parameters are positive.

TheOREM 1. The set $\mathcal{S}$ is positively invariant for the systems described by (9a) and (9b).

Proof. Due to the positivity of $\dot{T}$ for every $x \in\{(T, V, I, U): 0<T<$ $\left.T_{\max }, V \geq 0, I \in[0,1], U \in \mathbb{R}\right\}$, no state with $T \leq 0$ can be reached from $\mathcal{S}$ by any solution to $(9 \mathrm{a})$ or $(9 \mathrm{~b})$.

Moreover, for every state from $\mathcal{S}$ with $V=0$, we have $\dot{V}=0$. Therefore, no state with $V<0$ can be reached from $\mathcal{S}$.

Similarly, no state with $I \notin[0,1]$ can be reached from $\mathcal{S}$ by any solution to $(9 \mathrm{a})$ or $(9 \mathrm{~b})$. This is caused by the identity $\dot{I}=0$ at all points from $\mathcal{S}$ for which $I=0$ or $I=1$.

Additionally, there are no limitations on $U$, which together with the previous conclusions implies that $\mathcal{S}$ is invariant for the systems described by (9a) and (9b).

Theorem 2. For every $x^{*} \in \mathcal{S}$ the systems described by (9a) and (9b) have a unique solution through $x^{*}$ for every $t \geq 0$.

If the last coordinate of $x^{*} \in \mathcal{S}$ is positive, i.e. $U(0)>0$, then the solution to $(9 \mathrm{~b})$ is defined for every $t \geq 0$. If additionally the first coordinate is uniformly bounded, i.e. there exists $\bar{T} \geq T_{\max }$ such that $T(t) \leq \bar{T}$ for $t \geq 0$, then the solution to (9a) is defined for every $t \geq 0$.

Proof. The vector field $\Phi(T, V, I, U)$ of the system described by (9a) or $(9 \mathrm{~b})$ is of class $C^{\infty}$ in the whole set $\mathcal{S}$. Hence, every $x^{*} \in \mathcal{S}$ has a neighbourhood $\mathcal{Q}$ for which $\sup _{x=(T, V, I, U) \in \mathcal{Q}}|\Phi(x)|=M$ and $\Phi$ satisfies the Lipschitz condition with respect to $(T, V, I, U)$. From the Picard-Lindelöf theorem (see e.g. [7]) one gets the existence of a local solution to (9a) and (9b).

To prove the global (for $t \geq 0$ ) existence we show that all coordinates of the solution and their derivatives are bounded by some smooth functions of $t$ for all $t \geq 0$. 
We start our analysis from (9b). Positivity of $T$ and $V\left(\varepsilon_{1}-\gamma_{1} I\right)$ implies that $\dot{T} \leq \lambda_{1} T$. Therefore, $T(t) \leq T(0) \exp \left(\lambda_{1} t\right)$ for every $t \geq 0$.

For every state from $\mathcal{S}$ we have $\dot{U} \leq \lambda_{4} T$, which yields

$$
U(t) \leq U(0)+\frac{\lambda_{4}}{\lambda_{1}} T(0) \exp \left(\lambda_{1} t\right) \leq C_{U} \exp \left(\lambda_{1} t\right),
$$

where $C_{U}=U(0)+\frac{\lambda_{4}}{\lambda_{1}} T(0)$.

Assume now that $U(t)>0$ for every $t \geq 0$. Therefore, from the second equation of $(9 \mathrm{~b})$ one gets

$$
\dot{V} \leq \lambda_{2} C_{U} \exp \left(\lambda_{1} t\right) V, \quad \text { so } \quad V(t) \leq V(0) \exp \left(A e^{\lambda_{1} t}\right) \quad \text { for } A=\frac{\lambda_{2}}{\lambda_{1}} C_{U} .
$$

From the last equation of (9b) we obtain $\dot{U} \geq-\lambda_{5} V$, and hence

$$
U(t) \geq U(0)-c_{U} \exp \left(A e^{\lambda_{1} t}\right) \quad \text { with } \quad c_{U}=\frac{\lambda_{5} V(0)}{\lambda_{1} A} .
$$

To get an estimate for $\dot{I}$ one needs to estimate $X=V / T$. If $T$ is bounded away from 0 , e.g. $T \geq T_{\max }$, then it is easy to see that

$$
X(t) \leq \frac{V(0)}{T_{\max }} \exp \left(A e^{\lambda_{1} t}\right) .
$$

If $T<T_{\max }$, then estimating $\dot{X}$ we obtain

$$
\dot{X}=\left(\lambda_{2} U(1-I)-\lambda_{1}\left(1-\frac{T}{T_{\max }+V\left(\varepsilon_{1}-\gamma_{1} I\right)}\right)\right) X \leq \lambda_{2} U X,
$$

which, just as for $V$, implies $X(t) \leq C \exp \left(B e^{\lambda_{1} t}\right)$ for some $B, C>0$. Thus, in both cases $X(t) \leq C_{X} \exp \left(B_{X} e^{\lambda_{1} t}\right)$, where $B_{X}=\max \{A, B\}$ and $C_{X}=$ $\max \left\{C, V(0) / T_{\max }\right\}$. Finally,

$$
\begin{aligned}
-\lambda_{3} c_{U} \exp \left(A e^{\lambda_{1} t}\right)\left(C_{X} \exp \right. & \left.\left(B_{X} e^{\lambda_{1} t}\right)\right)^{\alpha} \\
& \leq \dot{I} \leq \lambda_{3} C_{U} \exp \left(\lambda_{1} t\right)\left(C_{X} \exp \left(B_{X} e^{\lambda_{1} t}\right)\right)^{\alpha} .
\end{aligned}
$$

The above inequalities show that in the set $\mathcal{S}$ until $U$ is positive the variables $T, V, I, U$ of (9b) and their derivatives are bounded by smooth (in fact at most double exponential) functions of $t$. On the other hand, if there exists $\bar{t}>0$ such that $U(\bar{t})=0$ and $U(t)<0$ in the right-hand side neighbourhood of $\bar{t}$, then $V$ and $I$ decrease (or do not change) until $U<0$, and hence $V(t) \leq V(\bar{t})$ and $I(t) \leq I(\bar{t})$ for $t$ in this neighbourhood. Therefore, the estimations obtained can be easily extended for such $t$. Hence, every solution in $\mathcal{S}$ with $U(0)>0$ is defined for every $t \geq 0$.

To prove global existence for (9a) we assume that $T(t) \leq \bar{T}$ for $t \geq 0$. Then $\dot{U} \leq \lambda_{4} \bar{T}$ and hence $U(t) \leq U(0)+\lambda_{4} \bar{T} t$, so $U(t) \leq \bar{C}_{U} \exp (A t)$ for some $A, C_{U}>0$. Further estimations are exactly the same as in the case of $(9 \mathrm{~b})$. 
It can be easily seen that for any parameter values there always exists a steady state. However, it is in a 2-dimensional manifold:

$$
\varphi(v, u)=\left(T_{\max }+v\left(\varepsilon_{1}-\gamma_{1}\right), v, 1, u\right),
$$

where $(v, u) \in[0, \infty) \times \mathbb{R}$. Moreover, for $1>\frac{\varepsilon_{1} \lambda_{4}-\lambda_{5}}{\gamma_{1} \lambda_{4}}$ there exists another manifold consisting of steady states, which is the line:

$$
\psi(s)=\left(\frac{T_{\max } \lambda_{5}}{\lambda_{5}-\lambda_{4} \varepsilon_{1}+\lambda_{4} \gamma_{1} s}, \frac{T_{\max } \lambda_{4}}{\lambda_{5}-\lambda_{4} \epsilon_{1}+\lambda_{4} \gamma_{1} s}, s, 0\right),
$$

where $s \in[0,1] \cap\left(\frac{\varepsilon_{1} \lambda_{4}-\lambda_{5}}{\gamma_{1} \lambda_{4}}, \infty\right)$. Therefore, we can expect that depending on the initial values, the solutions can tend to different steady states.

3. Numerical simulations of tumour growth. This section presents the results of numerical simulations of the systems (9a) and (9b). In the simulations we have used the parameter values estimated in [32] (cf. also Appendix at the end of this paper). The following parameters are fixed for all simulations:

$$
T_{\max }=3, \lambda_{1}=0.69, \beta_{1}=0.192, \varepsilon_{1}=4, \gamma_{1}=2, \lambda_{2}=0.5, \alpha=2 .
$$

We vary the remaining parameters $\lambda_{3}, \lambda_{4}$ and $\lambda_{5}$. The initial conditions are fixed for all simulations:

$$
T_{0}=3, \quad V_{0}=0.1, \quad I_{0}=0.001, \quad U_{0}=0.01 .
$$

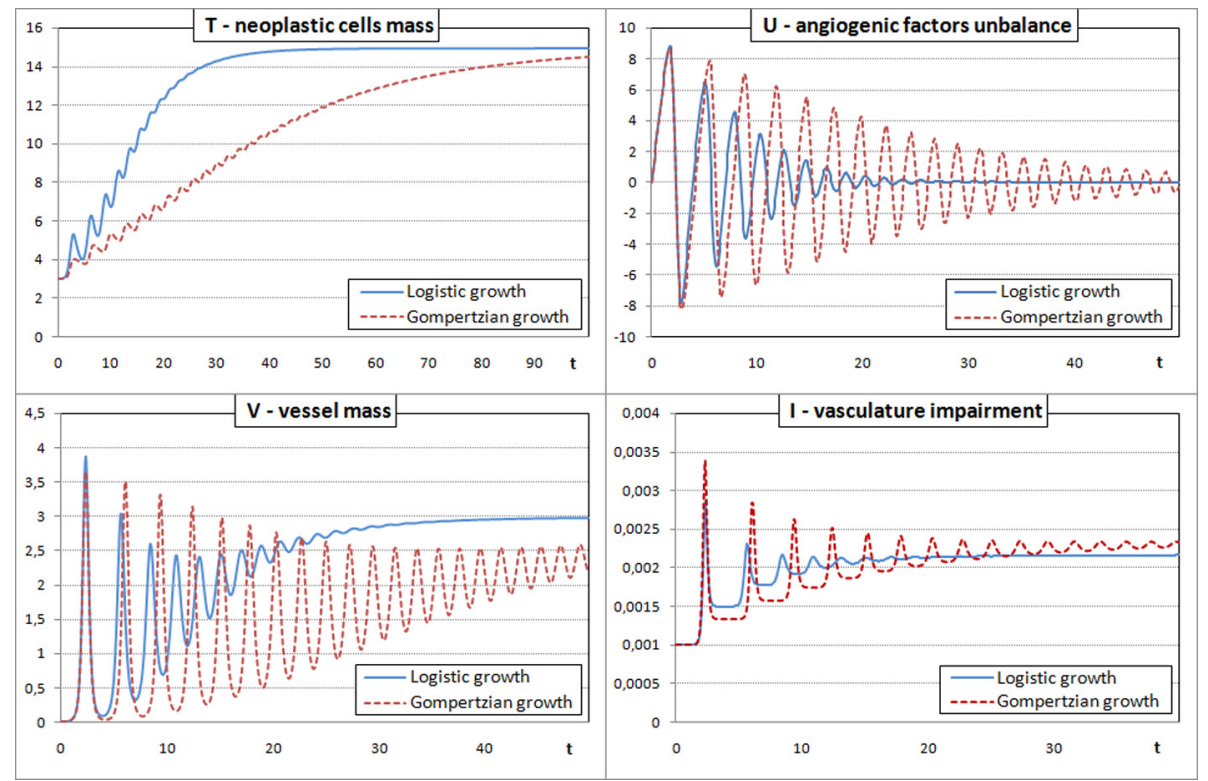

Fig. 1. Solutions to (9a) and (9b) for $\lambda_{3}=1, \lambda_{4}=2$ and $\lambda_{5}=10$ 
In Figures 1-4 we see the comparison between the dynamics of solutions to $(9 \mathrm{a})$ and $(9 \mathrm{~b})$. Every figure consists of four graphs which compare the behaviour of coordinates of both systems.

The solutions in Fig. 1 show that a dormant state of tumour can be reached due to high secretion of inhibitors during the vascularisation process. A high secretion rate of natural inhibitors also leads to the oscillatory behaviour of solutions. Convergence of solutions to the steady state is caused by dumping oscillations of $U$ around zero. This case shows that solutions tend to a positive steady state with oscillatory behaviour. A low level of impairment during the whole vascularisation process indicates that the tumour has proper vessel structure. Thus, this type of tumour should be sensitive to anti-angiogenic treatment and chemotherapy. We see that the Gompertzian type of tumour growth causes slower dynamics of this growth and higher amplitudes of oscillations compared to the logistic type of growth. Both solutions tend to some steady states with similar values of variables.

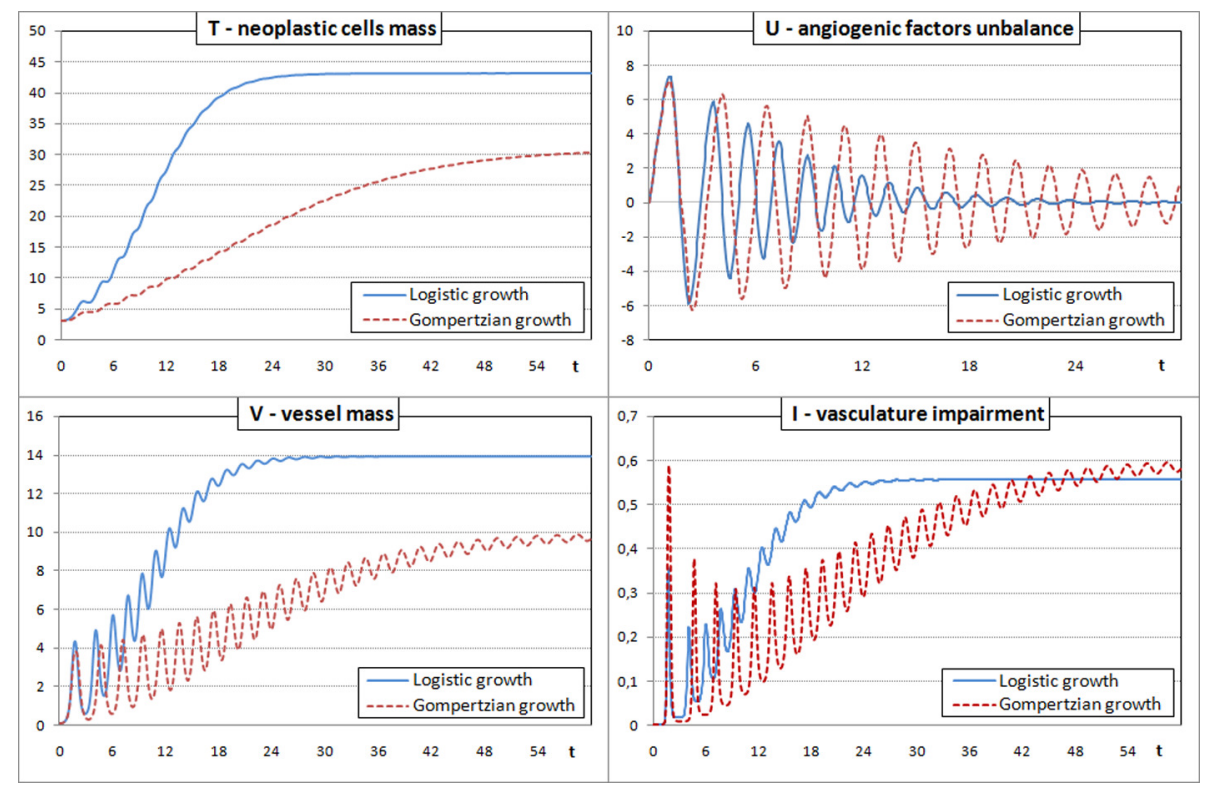

Fig. 2. Solutions to (9a) and (9b) for $\lambda_{3}=5, \lambda_{4}=2.9$ and $\lambda_{5}=9$

The solutions in Fig. 2 also show convergence to the steady state caused by dumping oscillations of $U$ around zero, but impairment stabilizes on a much higher level. Again, in the solution with the Gompertzian equation, slower dynamics of the tumour growth is observed. However, the two solutions differ much in the tumour mass of the steady state reached. 


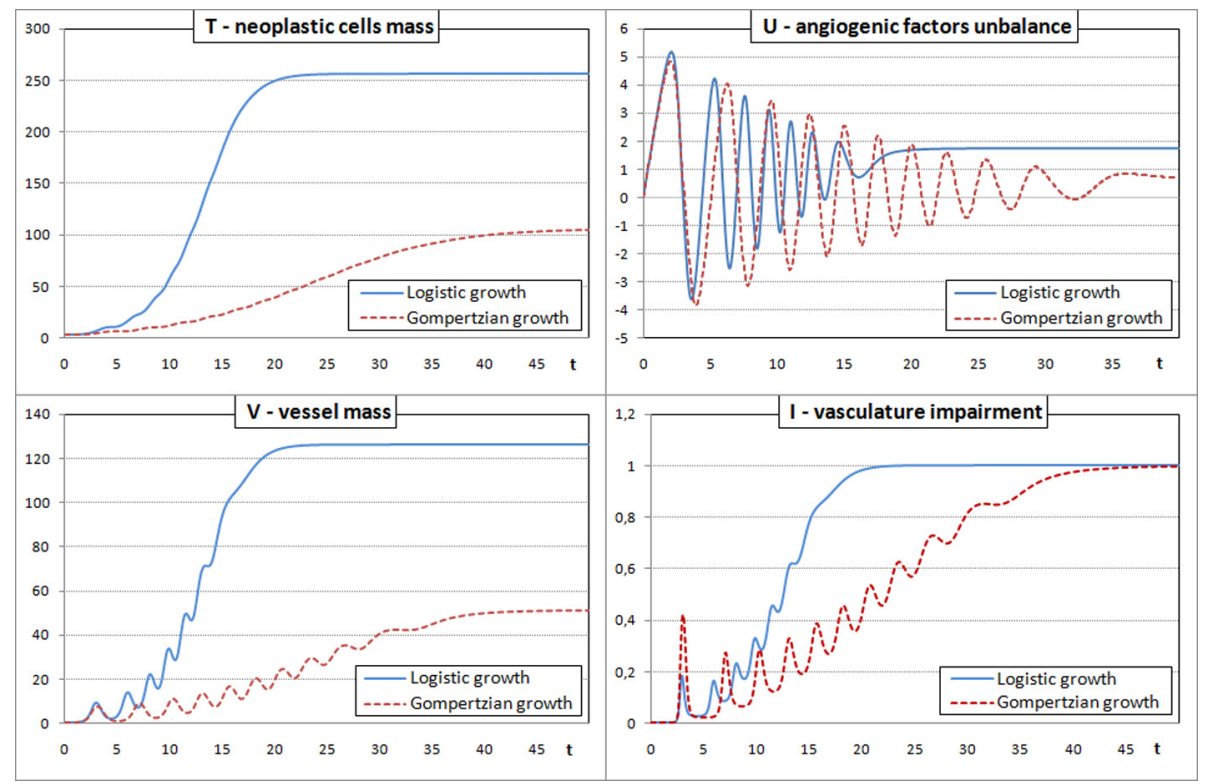

Fig. 3. Solutions to (9a) and (9b) for $\lambda_{3}=1.7, \lambda_{4}=1$ and $\lambda_{5}=2$

In simulations presented in Fig. 3 we can also see that the solutions tend to a positive steady state. However, in this case the dormant state is

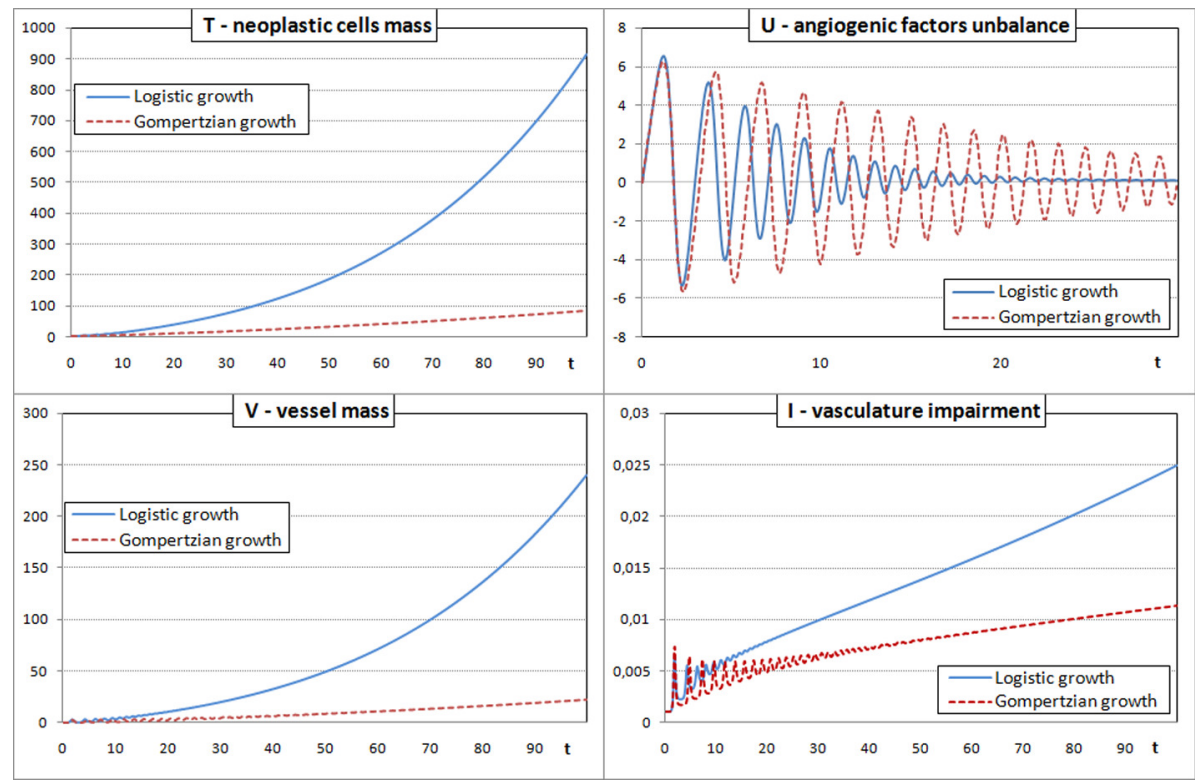

Fig. 4. Solutions to (9a) and (9b) for $\lambda_{3}=2.7, \lambda_{4}=2.6$ and $\lambda_{5}=9.9$ 
attained due to the high level of vasculature impairment that tends to its greatest value equal to 1 . By changing the parameter values the same type of convergence to the steady state can be achieved but with strictly increasing values of each coordinate. The high level of impairment indicates that the tumour has incorrect and highly inefficient vessel structure. Thus, in this type of tumour growth, lowest efficiency of treatment is expected. Solutions to $(9 \mathrm{a})$ and $(9 \mathrm{~b})$ differ in tumour growth dynamics and the steady state values reached.

Simulations in Fig. 4 show a prolonged exponential growth of tumour. It seems that the growth is unlimited, but the graph of the vasculature impairment $I$ suggests that both solutions should reach a steady state in finite time. In this case the difference between the two types of growth function is the most pronunced.

Summarising the results of simulations, similarly to [32] we can state the following:

COROllary 3. Independently of the assumed type of neoplastic cell dynamics, a vascular dormant state of tumour can be achieved in two different ways: due to almost $100 \%$ impairment of vasculature, that is, due to $I \rightarrow 1$ and $I \approx 1$, or due to dumping oscillations of unbalance around zero with $U \rightarrow 0$.

It should be remarked that each case is different in the final level of impairment and unbalance, so treatments should differ in efficiency. It can also be seen that oscillatory behaviour of the state variables is very dominant in the simulations. This is caused by reaction of the host tissue against too high proangiogenic factor level and vessel mass. Because of higher secretion of natural angiogenic inhibitors, for short periods of time the unbalance $U$ is brought down below zero. This causes a temporary degradation of newly formed vessels and therefore a temporary decrease in tumour mass. By changing the values of parameters so that the reaction of the host tissue is weak from the beginning of tumour angiogenesis, all oscillations can be dumped.

4. Chemotherapy and antiangiogenic treatment. To include antiangiogenic therapy in the model, we modify the last equation of (9) to

$$
\dot{U}=\left(\lambda_{4} T-\lambda_{5} V-\lambda_{6} g_{a}(t)\right)(1-I) .
$$

The function $g_{a}(t)$ represents the amount of inhibitors administered at a given time. It generally includes partially cleared contributions from prior administration at earlier times $t^{\prime}<t$. Under the usual pharmacokinetic assumptions, we use the following expression for $g_{a}(t)$ (see [19]): 


$$
g_{a}(t)=\int_{0}^{t} c_{a}(s) \exp \left(-\theta_{a}(t-s)\right) d s
$$

where $c_{a}(s)$ is the rate of administration of the inhibitor concentration at time $s$ and $\theta_{a}$ is the clearance rate. We assume that the inhibitors are administered as boli, so we use the density of normal distribution to determine the function $c_{a}(s)$. Let $t_{1}^{a}, \ldots, t_{n}^{a}$ be the sequence of time instances at which the inhibitors are administered. We propose the following equation for $c_{a}(s)$ :

$$
c_{a}(s)=D_{a} \sum_{i=1}^{n} \frac{1}{h \sqrt{2 \pi}} \exp \left(-\frac{\left(s-t_{i}^{a}\right)^{2}}{2 h^{2}}\right),
$$

where $h$ is some small positive parameter and $D_{a}$ is the administered dose of inhibitors.

Chemotherapy effectiveness is highly dependent on the possibility of its even distribution in all tumour regions. Thus, a higher ratio of vessel mass to neoplastic cell mass implies higher effectiveness. Simultaneously, a higher level of vessel impairment yields lower quality of matter distribution. Hence, chemotherapy is incorporated in the following change in the first equation of (9):

$$
\dot{T}=T h\left(1-\frac{T}{T_{\max }+V\left(\epsilon_{1}-\gamma_{1} I\right)}\right)-\tau_{1} T\left(1+\frac{V}{T}\left(\tau_{2}-\tau_{3} I\right)\right) g_{c}(t),
$$

where $\tau_{1}>0$ and $0<\tau_{3}<\tau_{2}$. The function $g_{c}(t)$ is determined in the same way as the function $g_{a}(t)$, so it has the form

$$
g_{c}(t)=\int_{0}^{t} D_{c} \sum_{i=1}^{n} \frac{1}{h \sqrt{2 \pi}} \exp \left(-\frac{\left(s-t_{i}^{c}\right)^{2}}{2 h^{2}}\right) \exp \left(-\theta_{c}(t-s)\right) d s .
$$

Exemplary plots of $g_{a}(t)$ are presented in Fig. 5 .

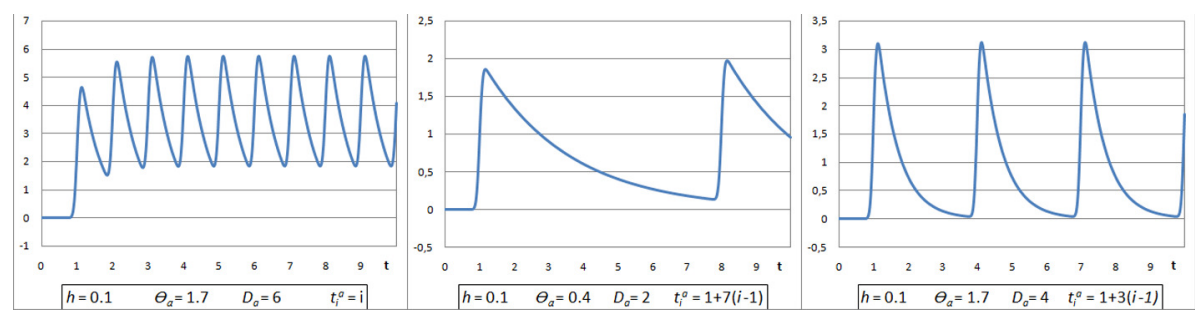

Fig. 5. Exemplary plots of $g_{a}(t)$

4.1. Numerical simulations of tumour treatment. This section presents the results of numerical simulations of the system described by (9a) with different types of treatment. Results for (9b) with the logistic type of tumour growth are qualitatively the same. However, they can be obtained for 


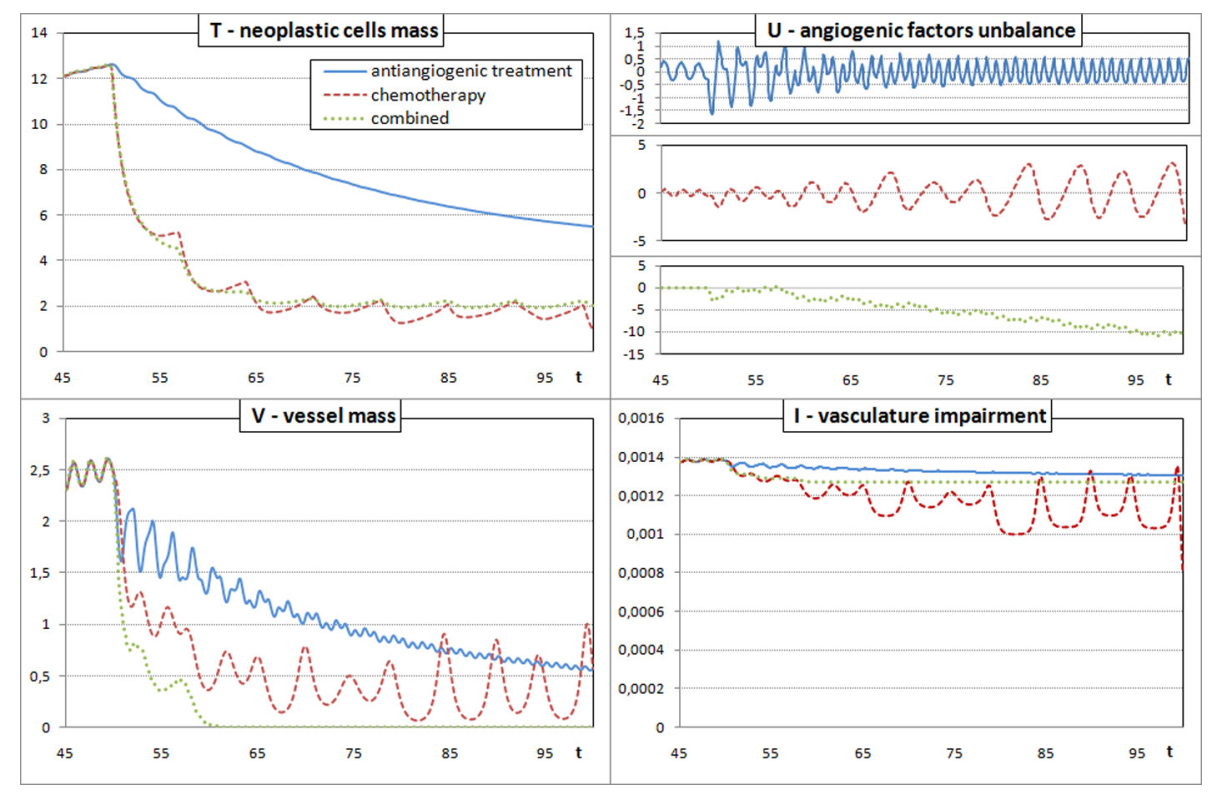

Fig. 6. Solution to (9a) for the parameters as in Fig. 1 under different treatment conditions. Dose $D_{a}=9$ of inhibitors is administered from time $t_{1}^{a}=50$ on. Dose $D_{c}=2$ of chemotherapy is administered from time $t_{1}^{c}=50 \mathrm{on}$.

different parameter values than those used in simulations of (9a). Some of the results for $(9 \mathrm{~b})$ were presented in [32].

In view of current methods of treatment the interval between each administration of inhibitors is set to one day for each simulation. For chemotherapy it is set to one week. The following parameters are fixed for all simulations:

$$
h=0.1, \quad \theta_{a}=1.7, \quad \theta_{c}=0.4 .
$$

We change only the dose and the time of first administration of drugs.

The solution in Fig. 6 shows that in the case of a tumour dormant state with low vasculature impairment, every treatment gives good results in tumour mass reduction. The graphs also show that a combined therapy does not improve the treatment results significantly. Slightly better results occur only at the beginning, and what is more important, the simulations reveal that removing all vessels by administering angiogenic inhibitors leads to a decrease of chemotherapy effectiveness. It seems that in this case it is better to use chemotherapy only.

A qualitative change of effectiveness can be seen in Fig. 7. In the case of tumour dormancy caused by the full vasculature impairment, only a combined therapy reduces the tumour mass effectively. However, as Fig. 7 shows, better results of a combined treatment may occur with some delay. A high dose of angiogenic inhibitors (compared to the previous case) does not even 


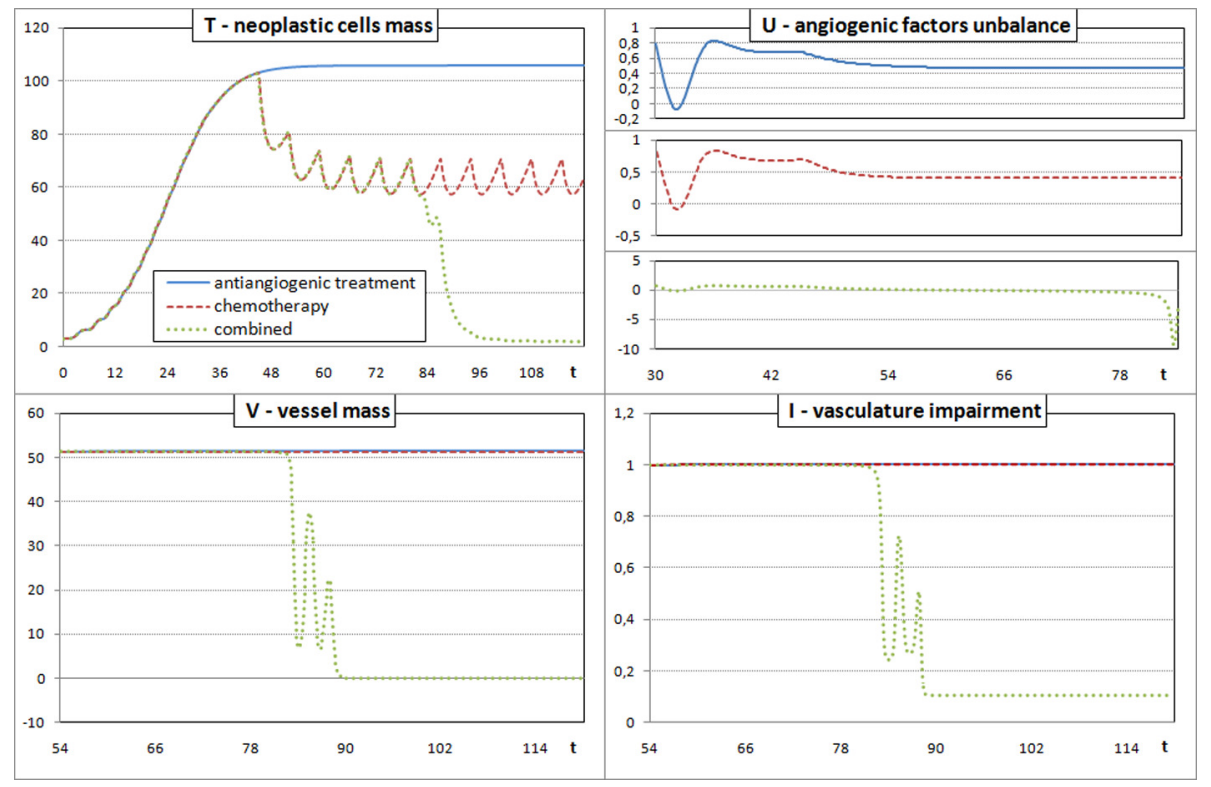

Fig. 7. Solution to (9a) for the parameters as in Fig. 3 under different treatment conditions. Dose $D_{a}=20$ of inhibitors is administered from time $t_{1}^{a}=45$ on. Dose $D_{c}=2$ of chemotherapy is administered from time $t_{1}^{c}=45$ on.

stop the tumour growth. Administering chemotherapy weekly causes only a temporary and insignificant tumour mass reduction.

The simulation in Fig. 8 shows that in the case of persistent exponential growth of tumour, a much higher dose of angiogenic inhibitors than in the first case is needed to successfully reduce the tumour mass, even though impairment is at a similar level. Nevertheless, the low level of vessel impairment and the high ratio of vessel mass to neoplastic cell mass during the whole growth allows chemotherapy reach high effectiveness. Combining angiogenic inhibitors with chemotherapy gives similar results as in the case of a dormant state with low vessel impairment.

In Fig. 9 an unfavourable effect of antiangiogenic treatment is presented. Administering a too small dose of angiogenic inhibitors changes the behaviour of the solution presented in Fig. 3. Due to that change the tumour reaches another dormant state with higher mass.

5. Summary. We have proposed two models of tumour growth described by systems of four ODEs that involve neoplastic cell mass $T$, vessel mass $V$, level of vessel impairment $I$ (measured as the percentage of vessels impaired) and angiogenic factor unbalance $U$ (measured as the difference between stimulators and inhibitors). The models proposed differ in the type of neoplastic cell dynamics assumed. We have assumed either the logistic or 

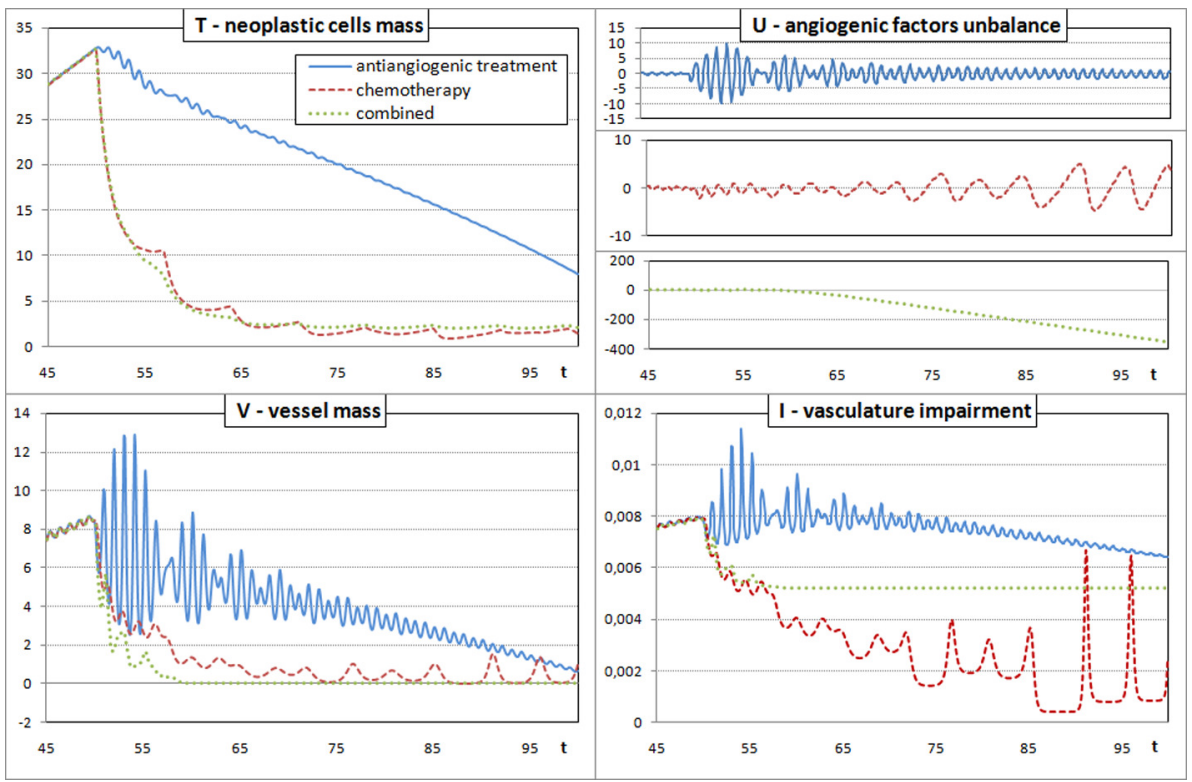

Fig. 8. Solution to (9b) for the parameters as in Fig. 4 under different treatment conditions. Dose $D_{a}=25$ of inhibitors is administered from time $t_{1}^{a}=50$ on. Dose $D_{c}=2$ of chemotherapy is administered from time $t_{1}^{c}=50$ on.
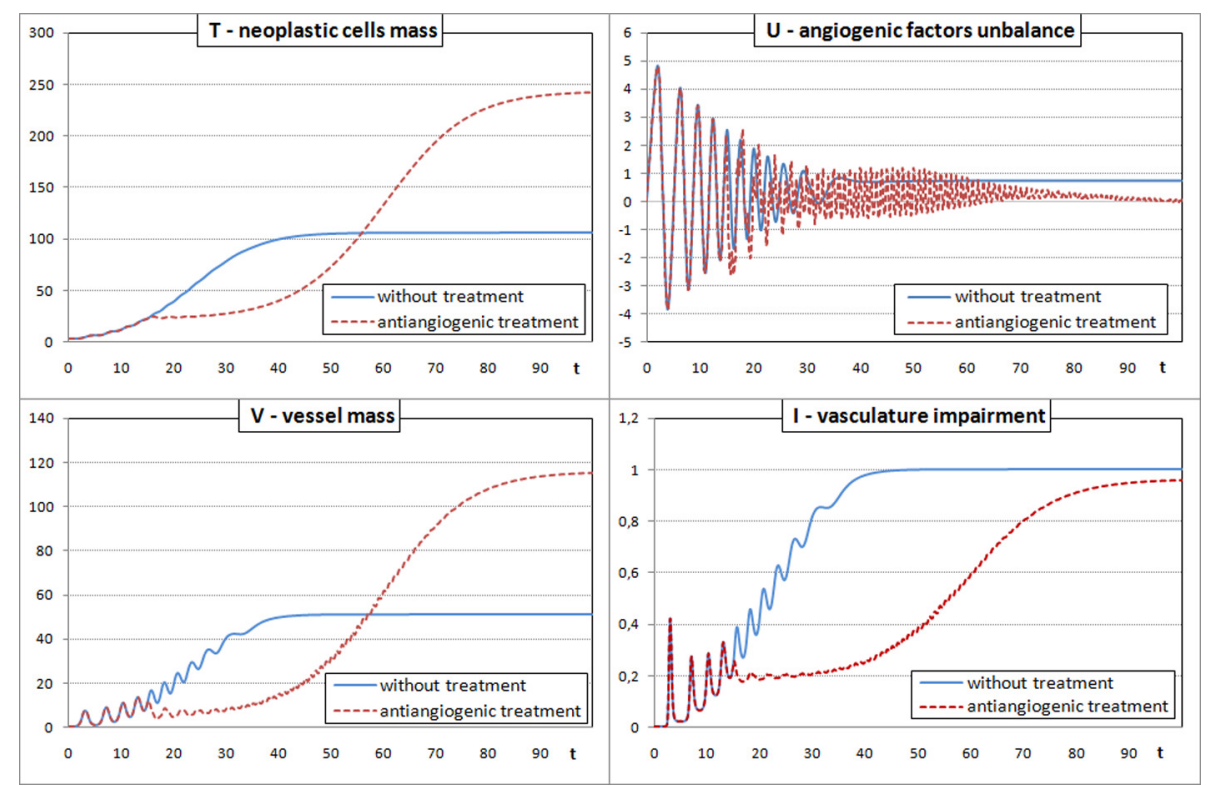

Fig. 9. Unfavourable influence of antiangiogenic treatment on the solution to (9a) for the parameters as in Fig. 3. Dose $D_{a}=20$ of inhibitors is administered from time $t_{1}^{a}=15$ on. 
Gompertzian type of growth. We have mainly focused on the comparison between the dynamics of both systems. In both models three types of treatment have been included: standard chemotherapy, antiangiogenic therapy and a combined treatment.

In Section 1 we have explained the process of vessel impairment, while in Section 2 the models have been proposed. Moreover, in Subsection 2.1 some basic mathematical properties of the models have been described. It has been proven that for every $x$ in the invariant set found, there exists a unique solution to both systems through $x$ and under some conditions it exists globally in time (for $t \geq 0$ ). Two different sets of steady states have been found and only one of them exists independently of the parameter values.

The numerical simulations of growth in Section 3 show that a dormant state of tumour can be reached in two different ways, either due to increasing the vessel impairment to its maximal level equal to 1 , or due to dumping oscillations of the unbalance between stimulators and inhibitors. These types of tumour dynamics do not depend on the type of growth assumed. All the types of outcomes obtained for $(9 \mathrm{~b})$ and presented in [32] can also be obtained for $(9 \mathrm{a})$. Therefore, the qualitative behaviour of both systems described by (9a) and (9b) is very similar. However, there are quantitative differences - the same type of behaviour has been found for different parameter values.

Section 4 presents results for three types of treatment for (9a). These results are very similar to those obtained in [32] for (9b). We have compared three types of treatment: anti-angiogenic therapy, that is, administration of inhibitors of the angiogenesis process, chemotherapy and a combined therapy. Numerical simulations show that in each case different qualitative results occur. It should be remarked that although a combined therapy seems to be better in most cases, there are parameter values for which simulations suggest using only chemotherapy. On the other hand, it may also happen that the influence of anti-angiogenic treatment occurs with some delay. This suggests that even if at the beginning the combined therapy is not more efficient than chemotherapy, it can become so after some time. It should also be remarked that even if the combined therapy seems to give better results, administration of angiogenic inhibitors should be stopped at some time, when the impairment reaches a sufficiently low level. The low level of impairment means that the vessel structure has been improved during the drug administration, and better vessel structure can help in better chemotherapy administration.

6. Appendix. In this appendix, following [32] we explain estimation of some parameter values for (9). We use gram as mass unit and day (average time of cell division) as time unit. 
At the beginning of avascular tumour growth, when its mass is small, the number of its cells should approximately double each day. This yields the estimate $\lambda_{1}=\log (2) \approx 0.69$. Following Hahnfeldt et al. [19] the value of $\beta_{1}$ is fixed at 0.192 . We assume that one gram of vessels can support four grams of neoplastic cells $\left(\varepsilon_{1}=4\right)$ and full impairment causes a decrease of vessel efficiency by half $\left(\gamma_{1}=2\right)$. Suppose $\lambda_{2}=0.5$, because the vessel growth dynamics is lower than tumour growth.

Therefore, the following parameters are fixed for all simulations:

$$
T_{\max }=3, \quad \lambda_{1}=0.69, \quad \beta_{1}=0.192, \quad \varepsilon_{1}=4, \quad \gamma_{1}=2, \quad \lambda_{2}=0.5 .
$$

Also the initial conditions are fixed for all simulations:

$$
T_{0}=3, \quad V_{0}=0.1, \quad I_{0}=0.001, \quad U_{0}=0.01 .
$$

The initial condition $T_{0}=T_{\max }=3$ reflects the fact that in most cases the tumour gets the ability to secrete proangiogenic factors after being in the avascular dormant state for some time. This state is achieved for maximal avascular tumour size $T_{\max }$. Values of the other initial conditions mean that tumour angiogenesis has started, especially that proangiogenic factors are secreted, which is reflected by $U_{0}>0$.

\section{References}

[1] B. Alberts, A. Johnson, J. Lewis, M. Raff, K. Roberts and P. Walter, Molecular Biology of Cell, Garland Publ., New York, 2007.

[2] Z. Agur, L. Arakelyan, P. Daugulis and Y. Ginosar, Hopf point analysis for angiogenesis models, Discrete Contin. Dynam. Systems B 4 (2004), 29-38.

[3] L. Arakelyan, V. Vainstein and Z. Agur, A computer algorithm describing the process of vessel formation and maturation, and its use for predicting the effects of antiangiogenic and anti-maturation therapy on vascular tumor growth, Angiogenesis 5 (2002), 203-214.

[4] I. D. Bassukas, Comparative Gompertzian analysis of alterations of tumor growth patterns, Cancer Res. 54 (1994), 4385-4392.

[5] M. Bodnar and U. Foryś, Three types of simple DDEs describing tumour growth, J. Biol. Systems 14 (2007), 453-471.

[6] - - - Angiogenesis model with carrying capacity depending on vessel density, ibid. 17 (2009), 1-25.

[7] I. N. Bronshtein, K. A. Semendyayev, G. Musiol and H. Muehlig, Handbook of Mathematics, 5th ed., Springer, Berlin, 2007.

[8] Cancer Modeling and Simulation, L. Preziosi (ed.), Chapman \& Hall, 2003.

[9] A. d'Onofrio and A. Gandolfi, Tumour eradication by antiangiogenic therapy: analysis and extensions of the model by Hahnfeldt et al. (1999), Math. Biosci. 191 (2004), 159-184.

[10] - - - The response to antiangiogenic anticancer drugs that inhibit endothelial cell proliferation, Appl. Math. Comput. 181 (2006), 1155-1162.

[11] - - - A family of models of angiogenesis and antiangiogenesis anticancer therapy, Math. Med. Biol., to appear. 
[12] A. Ergun, K. Camphausen and L. M. Wein, Optimal scheduling of radiotherapy and angiogenic inhibitors, Bull. Math. Biol. 65 (2003), 407-424.

[13] J. Folkman, Tumor angiogenesis: Therapeutic implications, N. Engl. J. Med. 285 (1971), 1182-1186.

[14] - Agiogenesis in cancer, vascular, rheumatoid and other disease, Nat. Med. 1 (1995), 27-31.

[15] —, Angiogenesis, Ann. Rev. Med. 57 (2006), 1-18.

[16] U. Foryś and A. Marciniak-Czochra, Logistic equation in tumour growth modelling, Int, J. Appl. Math. Comput. Sci. 13 (2003), 317-325.

[17] S. A. Frank, Dynamics of Cancer-Incidence, Inheritance, and Evolution, Princeton Univ. Press, Princeton, 2007.

[18] B. Gompertz, On the nature of the function expressive of the law of human mortality, and a new mode of determining the value of life contingencies, Philos. Trans. Roy. Soc. 115 (1825), 513-585.

[19] P. Hahnfeldt, D. Panigrahy, J. Folkman and L. Hlatky, Tumor development under angiogenic signaling: a dynamical theory of tumor growth, treatment response and postvascular dormancy, Cancer Res. 59 (1999), 4770-4775.

[20] R. K. Jain, Taming vessels to treat cancer, Scientific Amer. 298 (2008), 56-63.

[21] - Normalization of tumour vasculature: an emerging concept in antiangiogenic therapy, Science 307 (2005), 58-62.

[22] A. K. Laird, Dynamics of tumour growth, British J. Cancer 18 (1964), 490-502.

[23] - Dynamics of tumour growth: comparison of growth rates and extrapolation of growth curve to one cell, ibid. 19 (1965), 278-291.

[24] U. Ledzewicz and H. Schättler, Optimal control for a system modeling tumor antiangiogenesis, in: Proc. ICGST Inter. Conf. Automatic Control and System Engineering, 2005, 147-152.

[25] - , - Antiangiogenic therapy in cancer treatment as an optimal control problem, SIAM J. Control Optim. 46 (2007), 1052-1079.

[26] - - - Analysis of a mathematical model for tumor anti-angiogenesis, Optim. Control Appl. Meth. 29 (2008), 41-57.

[27] - - - Optimal and suboptimal protocols for a class of mathematical models for tumor anti-angiogenesis, J. Theor. Biol. 252 (2008), 295-312.

[28] N. V. Mantzaris, S. Webb and H. G. Othmer, Mathematical modeling of tumorinduced angiogenesis, J. Math. Biol. 49 (2004), 111-187.

[29] J. D. Murray, Mathematical Biology I: An Introduction, Springer, New York, 2002.

[30] M. S. O'Reilly et al., Agiostatin: A novel angiogenesis inhibitor that mediates the suppression of metastases by a Lewis lung carcinoma, Cell 79 (1994), 315-328.

[31] - Endostatin: An endogenous inhibitor of angiogenesis and tumor growth, ibid. 88 (1997), 277-285.

[32] J. Poleszczuk, Tumor development model under angiogenic signaling with dependence on vessel impairment, in: Proc. National Conference on Application of Mathematics in Biology and Medicine, M. Bodnar and U. Foryś (eds.), Univ. of Warsaw, 2008, 104-109.

[33] A. Świerniak, Comparison of six models of antiangiogenic therapy, Appl. Math. (Warsaw) 36 (2009), 333-348.

[34] A. Świerniak, G. Gala, A. Gandolfi and A. d'Onofrio, Optimization of anti-angiogenic therapy as optimal control problem, in: Proc. 4th IASTED Conf. on Biomechanics, ACTA Press, M. Doblaré (ed.), 2006, 56-60. 
[35] A. Świerniak, A. d'Onofrio and A. Gandolfi, Control problems related to tumor angiogenesis, in: Proc. IEEE Industrial Electronics-IECON2006 (Paris 2006), 677681.

[36] T. E. Wheldon, Mathematical Models in Cancer Research, Hilger Publ., BostonPhiladelphia, 1998.

Institute of Applied Mathematics and Mechanics

Faculty of Mathematics, Informatics and Mechanics

University of Warsaw

Banacha 2

02-097 Warszawa, Poland

E-mail: urszula@mimuw.edu.pl

Received on 15.10.2008;

revised version on 9.3.2009 
